\section{$\underset{\substack{\text { hommes } \\ \text { \& migrations }}}{ }$}

\section{Hommes \& migrations}

Revue française de référence sur les dynamiques

migratoires

$1293 \mid 2011$

L'immigration dans les musées

\title{
Atone et aboulique
}

\section{Mustapha Harzoune}

\section{(2) OpenEdition \\ Journals}

\section{Édition électronique}

URL : http://journals.openedition.org/hommesmigrations/535

DOI : 10.4000/hommesmigrations.535

ISSN : 2262-3353

\section{Éditeur}

Musée national de l'histoire de l'immigration

\section{Édition imprimée}

Date de publication : 1 septembre 2011

Pagination : 142-147

ISSN : 1142-852X

Référence électronique

Mustapha Harzoune, "Atone et aboulique», Hommes \& migrations [En ligne], 1293 | 2011, mis en ligne le 18 février 2013, consulté le 20 avril 2019. URL : http://journals.openedition.org/

hommesmigrations/535; DOI : 10.4000/hommesmigrations.535 


\section{Kiosque}

\section{Atone et aboulique}

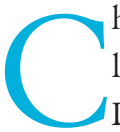

haque 14 juillet, la France exalte

la liberté et la concorde nationale.

Les uns célèbrent la prise de

la Bastille, "l'orage populaire" (Ilya Ehrenbourg) qui tonne pour faire tomber les bastilles qui continuent de se dresser sur le chemin de la liberté, les autres fêtent sous la forme d'une parade cordiale et militarisée la concorde et la fraternité républicaines. Chaque 14 juillet, deux années - 1789 et 1790 - s'enlacent, se congratulent, se jaugent et parfois se querellent. Ce 14 juillet, "la première personnalité française naturalisée à se présenter à une élection présidentielle française" (L'Humanité, 16 juillet) s'est crue autorisée à se mêler de l'intendance.

Badaboum! Tout ce que la France compte d'importants et de notabilités, de gras et de fats, s'est empressé de rappeler à la petite madame sa condition et ses origines.

Alors quoi ? Des Français de papiers se permettraient de donner des leçons aux nationaux pur sucre, aux Français "racinés", comme dirait Marcel Destienne, aux détenteurs d'une AOC sur leurs pièces d'identité ? Et c'est au nom de la République et de la nation, celle bien sûr d'Ernest Renan, que des lignes de démarcation sont tracées entre Français. "Lave les mots traînés dans la boue / et les bouches putrides", dit le poète Abdelatif Laâbi.

Ça gueule ! Ça hurle ! Ça vocifère !

Ça s'étouffe d'indignation! Ça invective et ça anathémise... au nom de quoi ? Au nom de la République bien sûr, de l'Histoire, de la Nation et du peuple - tout entier et sans distinguo. La malheureuse Eva n'émit qu'une suggestion, même pas une bombinette, un simple "rêve", même pas iconoclaste, car sur le sens du 14 juillet, les Français et les historiens balancent. En 1880, "L'Assemblée nationale $a(. .$.$) adopté le 14$ juillet comme fête nationale, mais sans préciser si elle se référait à 1789 ou 1790"(Le Figaro, 15 juillet). On emprisonne Marianne dans un corset idéologique prestement ficelé par des mains malhabiles et empressées ? Ce corset l'entrave, la comprime... Marianne n'étoufferait-elle pas?

\section{"Mais sur-le-champ on est refoulé par les agents"}

insi, donc, un matin ensoleillé
de juillet, un jour de fête et de
farniente, une de ces journées faites pour l'amour et l'amitié, voici qu'une femme, le visage lumineux, barré d'un regard bleu cerclé de rouge, s'égare entre les pattes de la soldatesque républicaine tout occupée à arpenter la "plus belle avenue du monde". Les pioupious défilent. À la tribune les politiques plastronnent. Eva Joly rêve : "J'ai rêvé que nous puissions remplacer ce défilé par un défilé citoyen où nous verrions les enfants des écoles, où nous verrions les étudiants, où nous verrions aussi les seniors défiler dans le bonheur d'être ensemble, de fêter les valeurs qui nous réunissent." Comme le rapporte le site 
de France-Soir, la ci-devant magistrate du pôle financier fit sa déclaration "à l'occasion d'un rassemblement d'associations syndicales, politiques et de résistants place de la Bastille". Place de la Bastille, tout un symbole. "Ce n'est pas des valeurs que nous portons, ajouta Eva Joly, je pense que le temps est venu de supprimer les défilés militaires du 14 juillet parce que ça correspond à une autre période."

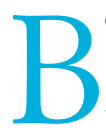
on! Même si on n'est pas là "pour regarder le defilé", "on est pas là [non plus] pour se faire engueuler", chantait Vian. Et comme dans la chanson, Eva Joly "sur-le-champ"a été "refoulée par les agents", autrement dit, ni une ni deux, les mamamouchis de l'ordre républicain ont fait la leçon à la petite dame, l'ont mise au piquet, affublée d'un bonnet d'âne et renvoyée à ses chères études - en Norvège ou ailleurs ! D’Abidjan, le Premier ministre a "réagi avec tristesse": "Je pense que cette dame n'a pas une culture très ancienne des traditions françaises, des valeurs françaises, de l'histoire française. (...) Si chaque année nous rendons hommage à nos forces armées le jour de la fête nationale, c'est parce que nous rendons hommage à une institution qui assure la défense des valeurs de la République française, de la liberté, de la fraternité, de l'égalité" (20minutes.fr, 15 juillet). Sur le même mode, le pitchoun Benjamin Lancar pour les Jeunes Populaires morigène son aînée : "Madame Joly n'a pas compris l'histoire de France et la signification du 14 juillet, soit elle cherche à provoquer et insulter nos militaires et les anciens combattants. Loin de la parade guerrière qu'elle dénonce, le 14 juillet est un grand moment de communion entre les Français et leur armée."Sur Europe 1, le 15 juillet, Henri Guaino a jugé cette proposition "pathétique":
"Dire ce qu'elle a dit c'est pour moi, profondément, une insulte à tous ceux qui depuis des siècles meurent pour ce pays, pour ses valeurs, pour sa liberté. (...) Elle rêve (...) de ce monde aseptisé et sans guerre dans lequel on a totalement occulté la dimension tragique de l'histoire. Je pense que c'est parfaitement irresponsable." Selon le conseiller du président, les propos d'Eva Joly trahiraient "une part d'incompréhension de ce qu'est la France". Pour Jean-Marie Bockel, ancien secrétaire d'État aux Anciens Combattants, "Eva Joly ne comprend rien à la sensibilité française et elle est totalement déconnectée de la réalité de notre pays. Madame Joly veut dénaturer une relation forte qui existe entre les Français et leur armée. (...) Elle pourrait être norvégienne et comprendre, mais elle ne comprend pas ou ne souhaite pas comprendre. Le reproche que je lui fais est de ne pas avoir saisi ce lien qui est au cceur de notre vivre ensemble. Elle est tout simplement amputée de cette sensibilité-là. Le patriotisme est un état d'esprit, et cet état d'esprit, Eva Joly ne l'a pas."

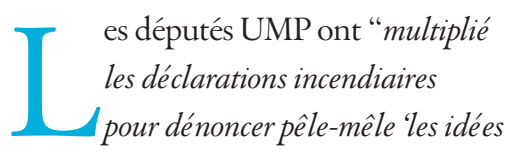
de soixante-huitarde attardée' et 'le pacifisme béat"' d'Eva Joly (Jacques Myard), (...) "un antimilitarisme que l'on croyait disparu avec les dernières communautés hippies"(Bruno Beschizza, Le Point.fr 16, juillet). Tout cela fut plaisant comparé aux déclarations du député UMP Guy Tessier, président de la commission de la défense à l'Assemblée nationale, qui épingla Eva Joly pour l'expédier dans le camp de l'“anti-France": "Je souhaiterais qu'un jour madame Eva Joly se trouve dans la situation de vos deux confrères que nos militaires sont allés libérer pour qu'elle trouve 
peut-être quelques vertus à nos soldats et à des défilés, fussent-ils guerriers. Je suis consterné d'entendre de telles déclarations, qui me rappellent les années d'avant la dernière guerre mondiale, où les pacifistes et les anti-militaristes nous ont amenés à cette triste défaite de 1940." (FranceInfo, 14 juillet). D'ailleurs, "l'esprit munichois souffle sur ces déclarations dégoulinantes de bêtise". "Comme certains fruits, certains politiques cachent sous la peau d'apparentes préoccupations environnementales la volonté gauchiste de détruire nos valeurs et nos institutions!' dixit le 15 juillet Christian Vanneste, député UMP du Nord, en comparant Eva Joly à "une pastèque”. De son côté, "Lionel Tardy, député UMP, a invité sur Twitter la député européenne à 'retourner en Norvège'.(...) Lionnel Luca, député chargé par l'UMP de conduire le débat sur la binationalité, s'interroge sur ce 'qu'elle aurait fait en 1944-1945"'(Les Inrocks.com 16 juillet). Marine Le Pen n'allait tout de même pas rater l'hallali. Elle a jugé "absolument consternants" les propos de l'ancienne juge d'instruction. Et dans son style inimitable: "Je ne crois pas qu'il soit légitime de se présenter à la présidence de la République quand on est devenu français tardivement et quand encore il y a quelques mois on était aux côtés du gouvernement norvégien pour lui apporter des conseils"(RTL, 15 juillet).

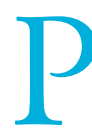

our Jean-Pierre Chevènement, "la nature de la France échappe sans doute" à Eva Joly. "Peut-être lui faut-il encore

un peu d'accoutumance", a-t-il ajouté (Le Parisien, 16 juillet). Le même jour dans le même quotidien, Daniel Cohn-Bendit ironise en demandant si on allait "reprocher à madame Carla Bruni-Sarkozy sa double nationalité. Parce qu'elle est franco-italienne, elle ne pourrait plus s'exprimer sur la France?"

\section{"On est pas là pour se faire engueuler"}

\author{
va Joly ne s'en est pas laissé conter. \\ L'ex-juge anticorruption a dispensé \\ quelques leçons républicaines.
}

Le 15 juillet sur Europe 1, elle répond : "Je ne descends pas de mon Drakkar, ça fait cinquante ans que je vis en France, donc je suis française." Le même jour sur le site du Point, elle sermonne: "prétendre défendre la République en agitant le démon des origines est une hypocrisie dangereuse". "Il [François Fillon] ferait mieux de relire La Révolution française de Michelet : la France républicaine est d'abord celle de ses citoyens et des valeurs qu'ils partagent. Elle n'est pas celle de leur lieu de naissance. (...) Un vrai républicain ne fait pas le tri entre les Français.”Le 16 juillet, sur le même site : "De tels excès n'étonnent guère quand ils proviennent des habituels matamores de l'identité nationale. Dans la bouche d'un Premier ministre, ils témoignent de la dégradation des termes du débat public et de la gangrène identitaire qui attaquent le corps républicain. Je ne permets pas que l'on mette en doute mon patriotisme. Je ne suis pas moins française que ceux qui me refusent le droit de m'exprimer."

La candidate aux présidentielles 2012 veut “que la République retrouve l'esprit de ses origines, car je suis préoccupée par le recul de l'idée républicaine dans notre pays. Sur cette vision, je ne pense pas être isolée. Ce sera l'un des enjeux de la campagne qui s'ouvre".

Dans Libération du 16 juillet, elle précise: "Je ne suis pas contre les défilés militaires en tant que tels. Mais le 14 juillet, ce n'est ni le lieu, ni le moment, pour ce genre de manifestation. Le 11 novembre ou le 8 mai sont beaucoup plus adaptés. (...) La République serait beaucoup 
mieux symbolisée par un défilé des personnes qui contribuent chaque année à entretenir le pacte républicain en travaillant au service de l'État ou dans des associations.” Pour Eva Joly, sa "binationalité n'est en rien un handicap. C'est une richesse culturelle que je partage avec un grand nombre de nos concitoyens. Les électeurs savent très bien que je suis française".

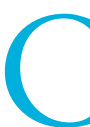
ôté commentaires des internautes, un certain "Carton jaune" rappelle que "dans les tranchées figuraient également nombre d'étrangers qui sont morts pour défendre des valeurs que représentait la France. D'autres ont créé des groupes de résistants et se sont sacrifiés pendant que certains Français servaient l'ennemi (...). Aujourd'hui leurs descendants sont traités de Français de seconde zone (...).Je crois qu'ils ont gagné notre reconnaissance, le droit de s'exprimer et de viore en paix chez eux car ils sont chez eux ne vous en déplaise" (Le Point.fr, 15 juillet). "Jeanne" écrit : "Pour moi comme pour beaucoup, ce jour est celui de la Révolution française et du meilleur qui en aura été retiré, droits et principes fondamentaux, liberté, égalité... Ce qui me dérange n'est pas la référence à l'histoire mais cette façon de condamner de facto et avec violence toute proposition, idée nouvelle et différence. Il n'y a pas de modération, de réflexion, d'intelligence dans les propos tenus en réaction. (...) La naissance et la position permettent-elles tout? Il y a haine (de l'autre) mépris (de la différence), bêtise dans les propos dommageables à l'image donnée du pays (...)."

\section{"Un vent mauvais souffle sur notre pays ${ }^{(1)}$ ')}

Et voilà qu'un élu de la République fait son mariole: "Afin di préparer la prochaine réunion UMP di la Colle qui devrait si tenir $l i$ lundi 26 septembre salle Paillere, ji vous propose étudier le programme di PS (...). Ji vous propose aussi un voyage di pèlerinage pour li 15 août à la mosquée di Strasbourg qui vient d'ouvrir, mesdames prévoyez un foulard. Ji vous souhaite un bon ramadan." Voilà la prose choisie par Gilbert Garelli, conseillé municipal UMP de la Colle-sur-Loup (Alpes-Maritimes), pour s'adresser à des militants UMP “indignés de l'humour anti-maghrébin" de l'élu. Malgré ses "excuses publiques" et ses "regrets", la presse s'est émue de "l'humour douteux" du conseiller (eMarrakech.info le 4 août).

Cet été le sport national consista à réexpédier ses concitoyens là d'où ils venaient.

Après Eva Joly, place à Jean-Vincent Placé. "Dans un entretien à Publicsénat.fr en marge de l'université d'été de l'UMP sur les prochaines élections sénatoriales, Alain Marleix, spécialiste de la carte électorale à l'UMP, déclare: 'Dans l'Essonne, [...] notre Coréen national, Jean-Vincent Placé, va avoir chaud aux plumes!"”

(Le Monde.fr, 3 septembre). Cela déplut au conseiller de Cécile Duflot : "C'est un scandale absolu, et je suis rarement scandalisé. Aujourd'hui il s'agit de moi, mais demain il dira de Manuel Valls 'notre Espagnol national' ou de Rachida Dati 'notre Marocaine nationale', ou encore de Rama Yade 'notre Sénégalaise nationale'. Le fait d'être coréen n'est bien sûr pas une infamie. Mais tout simplement, je suis français depuis trente-quatre ans et uniquement français. J'ai été adopté en 1975 par une famille normande, naturalisé deux ans plus tard, jai fait toute ma scolarité, mes études et toute ma vie professionnelle en France. J'ai travaillé à l'Assemblée nationale, je suis vice-président du conseil régional d'îlede-France et même chevalier de l'ordre national du Mérite (...) et tout ce que monsieur Marleix trouve à dire pour me qualifier c'est 'notre 
Coréen national. Cela démontre une fois de plus la lepénisation avancée de l'UMP(...).

Pour monsieur Marleix, manifestement, seul le sang donne le droit d'être français.

C'est une honte qu'un élu de la République puisse tenir de tels propos" (AFP). Le 5 septembre, sur France Info, Eva Joly réagit : "En fustigeant l'origine ou l'apparence de Jean-Vincent, on fustige en réalité tous les parents qui ont adopté en France, tous les enfants adoptés, et tous ceux qui ont un parent étranger, par exemple, tous les binationaux, et ceux-là finissent par faire des millions de Français."

\section{Menaces sur "le pacte démocratique"}

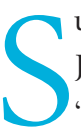
ur L'Express.fr, le 19 juillet, Jean-Yves Camus revient sur cette "contre-offensive basée sur le thème de lidentité et de la binationalité": "La droite tente d'instruire un procès à la gauche sur son supposé manque de patriotisme. C'est une constante du débat politique français depuis au moins un siècle et demi. Pourtant le patriotisme transcende les clivages politiques et n'a jamais été l'apanage de la droite. Il ne faut pas oublier que le patriotisme de la gauche a consolidé la République à la fin du XIX siècle. Il y eut ensuite l'Union sacrée en 1914, puis la Résistance qui a uni des patriotes de tous bords. Qui est patriote en 1871, Thiers ou les communards?' Pour le politologue, la droite bute sur un "problème de cohérence lorsqu'elle s'attaque au thème de l'identité nationale. En effet, chacun comprend quand la droite ditce qu'il ne faut pas être si l'on veut devenir Français : ne pas être un fondamentaliste musulman, un polygame, un individu refusant l'intégration... (...) Mais la frange la plus droitière de l'électorat veut bien plus : elle souhaite que certaines catégories d'étrangers, en raison de leur identité même, ne puissent plus devenir français. Selon eux, la nationalité ne doit plus s'acquérir par un acte de volonté mais seulement par la transmission. Or, pour aboutir à cela, il faudrait une révolution juridique qui constituerait une rupture majeure du pacte démocratique. L'UMP ne prendra pas ce risque mais elle ne peut pas le dire à ses électeurs droitiers. Pas plus qu'elle ne peut dire l'inverse aux centristes."

"La France, tu l'aimes ou tu la fermes !' écrit Rokhaya Diallo dans Le Monde du 6 août. Après avoir passé en revue les anathèmes de députés contre les rappeurs, et notamment ceux d'origine étrangère, les admonestations d'Éric Raoult à l'endroit de Marie N’Diaye, Goncourt 2009, les coups de semonce reçus par Eva Joly, l'auteure de Racisme, mode d'emploi (Larousse) tire le constat que "critiquer la France, lorsqu'on a des origines étrangères, une couleur de peau trop sombre ou un accent pas bien d'chez nous devient un exercice de plus en plus périlleux, c'est désormais un privilège octroyé aux seuls 'vrais' Français". Il fut un autre temps où des métèques, "de Brassens à Ferré, n’ont pas mâché leurs mots pour critiquer notre douce France. Mais loin d'être dénoncées par des députés en mal d'activité, leurs chansons sont passées à la postérité". Et de citer le Sétois aux origines italiennes: "Le jour du Quatorze Juillet, / Je reste dans mon lit douillet. / La musique qui marche au pas, / Cela ne me regarde pas."

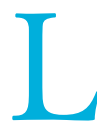

e 17 juillet sur Médiapart, Edwy Plenel cite Hugo qui, à la tribune du Sénat le 3 juillet 1880, déclare : "Messieurs, le 14-Juillet est une fête. (...) Cette fête est une fête populaire. Voyez la joie qui rayonne sur tous les visages, écoutez la rumeur qui sort de toutes les bouches. C'est plus qu'une fête populaire, c'est une fête nationale. Regardez ces bannières, entendez ces acclamations. C'est plus qu'une fête 
nationale, c'est une fête universelle. Constatez sur tous les fronts, anglais, espagnols, italiens, le même enthousiasme ; il n'y a plus d'étrangers."

\section{Sous le signe du métissage}

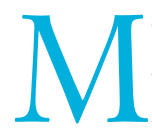
ais foin de considérations républicaines... le métissage ne ferait-il pas aussi partie de l'histoire de l'humanité ? Ainsi, JeuneAfrique.com (le 26 août) montre qu'aux USA, "hier, les métis passaient pour des traîtres. Ils sont aujourd'hui les hérauts d'une Amérique postraciale.(...) C'est la minorité arc-en-ciel de l'Amérique. Dans un pays où franchir la color line a longtemps été presque impossible - un véritable tabou -, beaucoup n'hésitent plus désormais à afficher leur origine multiethnique".

"Aux États-Unis, un mariage sur sept unit aujourd'hui des conjoints de races différentes. Un record. Certes, les Américains ne sont encore que 9 millions - soit 2,9\% de la population à se déclarer 'multiraciaux', selon la terminologie en vigueur ici, mais ils croissent et se multiplient à vitesse grand $V:+32 \%$ depuis 2000 , selon les chiffres du dernier recensement (2010)." Pour autant, "cette Amérique métisse a aussi ses détracteurs. (...) C'est moins au dépassement de la notion de race auquel nous assistons qu'à sa balkanisation, avec l'émergence d'une nouvelle tribu, celle des métis".

Le métissage souffle-t-il dans les pages du dictionnaire de l'Académie? Pas assez au goût de Pierre Assouline qui, le 4 août, sur son célèbre blog, "La République des lettres", salue l'arrivée d'Amin Maalouf à l'Académie française et y voit l'occasion d'anticiper "des progrès en arabe" de la docte institution. Il compte sur lui pour faire "avancer les travaux" et cite l'auteur des Identités meurtrières: "Lorigine de bien des mots est arabe, et cet apport mérite d'être mieux connu. (...) J'ai envie de faire un long travail là-dessus dont l'importance n'est pas seulement linguistique. Mettre en avant ces liens entre langues va au-delà de l'aspect linguistique et délivre un message d'une autre portée." Ce "message d'une autre portée" remonte loin dans l'histoire de l'humanité. Sur le site du Journal de la Science (le 6 septembre), on apprend qu'"une étude menée par le biologiste Michaël Hammer (université de Tucson, Arizona) suggère que Homo sapiens se serait métissé ponctuellement avec des Homo proches de sapiens, comme par exemple Homo erectus, au cours de ces 700000 dernières années. (...) Ce métissage aurait débuté sur le continent africain, très probablement avant les sorties du continent effectuées par Homo sapiens au cours des 200000 dernières années. (...)

Certes, on savait déjà, grâce aux travaux publiés en 2010par Svante Pääbo, que Homo sapiens s'était métissé avec son cousin Homo neandertal. Mais il s'agissait là d'un métissage s'étant produit hors d'Afrique (en effet, Homo neandertal n'a vraisemblablement jamais pénétré en Afrique)". Même si "seule l'analyse du génome complet des populations africaines viendra clore cette série d'hypothèses", dixit la généticienne Sarah Tishkoff (Nationalgéographic.fr 6 septembre), "cette découverte contredit le consensus classique selon lequel nous descendons tous d'une même population africaine, au matériel génétique 'pur'(...), explique Hammer, cité par le magazine Nature." Et pendant ce temps-là, Marianne, Marianne la républicaine, Marianne la métissée, ne devrait parler que d'une seule et autorisée voix. Atone et aboulique, Marianne.

\section{Mustapha Harzoune}

\section{Notes}

1. Eva Joly, Le Point.fr 15 juillet. 